\title{
Corps de la république. Blessure et maladie de M. Gambetta
} In: Genèses, 5, 1991. pp. 160-171.

Citer ce document / Cite this document :

Damamme Dominique. Corps de la république. Blessure et maladie de M. Gambetta. In: Genèses, 5, 1991. pp. $160-171$.

doi : $10.3406 /$ genes.1991.1085

http://www.persee.fr/web/revues/home/prescript/article/genes_1155-3219_1991_num_5_1_1085 


\title{
Corps
}

\section{de la République}

\author{
Blessure et maladie de M. Gambetta
}

\section{Dominique Damamme

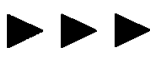

1. Odilon Lannelongue, Victor Cornil, « Blessure et maladie de M. Gambetta », in J. Reinach (éd.), Discours et plaidoyers politiques de M. Gambetta, 18\% vols., 1881-1885, vol. 9.

2.0. Lannelongue, professeur de pathologie à la iacl lté de médecine de Paris, président de 1. ssociation générale des médecins de France, ami foisonnel de Gambetta : Victor Cornil, également p...jesseur à la faculté de Paris, sénateur de l'Allier, alicien préfet gambettiste.

3. Le Concours médical, $\mathrm{n}^{\circ} 4,1883$. Sur le Concours, cf D. Damamme, «La jeunesse des syndicats de médecins ou l'enchantement du syndicalisme ", Genèses, nº 3 , mars 1991, p. 31-54.

4. «Blessure... », Paris, Glasson, 55 p.

5. Cf. le Roman vrai de la III et IV République, Paris, Bouquins, 1991 ; en particulier J. Reinach, « Le ministère Gambetta, histoire et doctrines (14 novembre 1881-26 janvier 1882) », 1884 et E. Pillias, « Léonie Léon, amie de Gambetta », 1935.

6. Elle préoccupe sérieusement les médecins : « L'état général du blessé demandant à être surveillé de près ; son embonpoint, son genre de vie réclamant quelques précautions », O. Lannelongue, op. cit., p. 11.

7. Les journalistes soulignent sa « fougue de Gascon et une franchise de gros garçon élevé un peu sommairement », « son habileté de Génois, mâtinée de judaïsme », son éloquence « à ventre déboutonné » (le Figaro, $1^{\text {cr }}$ janvier 1883 ) ; il est resté un « vieil étudiant pertubateur », un « personnage assez mal éduqué » (le XIX siècle, 3 janvier 1883). La Revanche ( 3 janvier 1883 ) voit en lui « un ventru et un viveur », et le Constitutionnel (3 janvier 1883), une « nature molle et sensuelle, affamée de jouissance ».

la différence des précédents « Documents » de Genèses, «Blessure et maladie de M. Gambetta ${ }^{1}$ » dont on pas un inédit. Avant de prendre place dans l'édition J. Reinach des discours de Gambetta, le rapport d'Odilon Lannelongue et de Victor Cornil $^{2}$ avait été publié dans la Gazette hebdomadaire et dans le Concours médical ${ }^{3}$, journaux médicaux liés aux républicains de gouvernement, puis, en brochure, chez Glasson $^{4}$. Unique en son genre depuis l'établissement de la démocratie parlementaire, ce texte s'impose à l'attention par la conjonction singulière d'un objet - l'agonie, "sur le vif », d'un grand homme - et d'une forme, le journal de bord d'un médecin.

Dans la journée du 27 novembre 1882, Léon Gambetta se blesse à la main en manipulant un revolver, point de départ du « drame des Jardies " ", sa maison de Ville-d'Avray. Il mourra le 31 décembre à minuit. Quelle que soit l'origine exacte de la blessure ${ }^{6}$ (maladresse ou querelle domestique qui tourne mal), il semble que Gambetta, dont le crédit est atteint par l'échec du "Grand ministère », ait immédiatement perçu les risques d'exploitation politique et s'en soit inquiété. En tout cas, dans un premier temps, l'homme politique préfère garder le silence et la confirmation officielle de la thèse de l'accident n'intervient qu'une semaine plus tard, pour couper court aux «bruits » intéressés qui se propagent dans la presse (la République française, 4 décembre 1882). Dans la conjoncture politique défavorable au gambettisme de l'année 1882, la nouvelle place les journalistes devant un dilemme : faut-il en parler? Et comment en parler? Son annonce s'étage ainsi sur plus d'une semaine. Elle figure dans l'édition du 29 novembre du Petit Parisien, mais le Petit Journal n'y fait écho que le 12 décembre. Certains ignorent ostensiblement l'information: ainsi le Figaro s'abstient-il de tout commentaire entre le $1^{\text {er }}$ et le 20 décembre. Jusqu'à 
l'ultime semaine de décembre, les journaux, dans leur grande majorité, se contentent de reprendre les communiqués de la République française, l'organe de Gambetta. Cette discrétion s'explique par des raisons très diverses, caractère bénin de la blessure, silence de l'entourage et des médecins, autorité mais aussi et inversement discrédit politique de Gambetta, prudence de la presse "indépendante ", indifférence des organes d'opposition, événements plus attractifs pour les feuilles populaires comme les inondations de la Seine, le procès de l'Union générale ou les préparatifs des fêtes de Noël. Évidemment, plusieurs quotidiens détonnent dans ce paysage hivernal, et d'abord l'Intransigeant de Rochefort qui, presque journellement, se sert de la blessure comme d'une arme, avec la violence qui lui est coutumière et distinctive, dans sa lutte contre le chef de l'opportunisme, tour à tour qualifié de «Machiavel cadurcien" (27 novembre 1882), d' «Ex-grand ministre», de "dictateur en retrait d'emploi », de «Génois néfaste " $\left(1^{\text {er }}\right.$ décembre 1882), de " cousin de $\mathrm{M}^{\text {lle }}$ Claire Gambetta », une chanteuse de music-hall qui passe au Bataclan (19 décembre 1882), ou mieux encore de "suppurant de la Ville-d'Avray » (idem) ${ }^{7}$. Très vite, se répand la rumcur d'une dispute entre Gambetta et sa maîtresse, "d'une violence inouïe " (l'Intransigeant, $1^{\text {er }}$ décembre 1882). "M. Gambetta ne s'est pas blessé. On a tiré sur M. Gambetta. Qui est on ? On est une femme. $\mathrm{M}^{\text {me }}$ L. X., la mère de l'enfant que $M$. Gambetta fait élever en Allemagne sous le nom de L. Massabié » (le Gaulois, «La vérité sur l'accident de Villed'Avray », 29 novembre 1882). "Ce ne serait pas un accident, et ce ne serait pas par luimême que M. Gambetta aurait été blessé. On fait remarquer à ce propos que l'événement a eu lieu à sa petite maison de Ville-d'Avray, qui n'est pas uniquement fréquentée, dit-on, par des hommes politiques. Faut-il croire qu'une de ces personnes étrangères à la politique [...] aurait reproché à celui-ci, le revolver à la main, de traiter certains serments comme un simple mandat de Belleville? " (l'Intransigeant, 30 novembre 1882). Les démentis de la République française n'y font rien ; « Les journaux ayant répandu toutes sortes de bruits sur la blessure de M. Gambetta, nous devons à nos lecteurs d'entrer dans des détails précis. M. Gambetta s'est blessé lui-même... » (la République française, 4 décembre 1882). « Ce bulletin mettra fin sans doute aux bruits divers et erronés qu'on répand sur cet accident » (idem, 5 décembre 1882). Même la connaissance de la nature réelle du mal dont est atteint Gambetta reste sans effet. "Comment une balle, reçue dans le bras, peut-elle occasionner une maladie dans le ventre. Nous allons l'expliquer : c'est que M. Gambetta n'a pas reçu une, mais deux balles [...] la seconde qui est entrée dans l'abdomen pour sortir près de l'aine » («La vérité sur le cas Gambetta», l'Intransigeant, 30 décembre 1882). Un des services espérés du rapport de Lannelongue sera de mettre définitivement fin à cette version des faits qui résiste à la mort. "Quelques journaux avaient affirmé que, indépendamment de la balle reçue dans le gras de la main droite et ressortie près du coude [...] M. Gambetta avait reçu une balle dans le ventre. Le procès-verbal d'autopsie n'en fait nullement mention; d'ailleurs cette version n'avait pas obtenu créance, mais on n'avait pas accepté le récit d'une blessure accidentelle en maniant un revolver. Tous les essais auxquels on s'est livré en ont démontré l'impossibilité. Ainsi que nous l'avons dit depuis le début, il y a eu drame. Seulement le blessé affirmant l'accident, sa déclaration est la seule officielle. Ce qui écarte d'ailleurs la pensée qu'il y ait pu y avoir crime prémédité, c'est que la personne qui s'est trouvée un revolver à la main, en présence de M. Gambetta, est restée à son chevet pendant tout le temps de sa maladie et l'a soigné avec un admirable dévouement (le Petit Journal, 3 janvier 1883).

Dans la dernière semaine de décembre, les journalistes qui s'alignaient sur l'optimisme 
de commande de la République française "On s'obstine à faire courir sur la santé de M. Gambetta des bruits qui sont faux ", (26 décembre 1882) - abandonnent leur réserve. «Il n'est plus temps maintenant d'équivoquer sur la santé de $\mathrm{M}$. Gambetta » (le Petit Journal, 31 décembre 1882). Le Temps dans son édition du 29 explique la différence entre la pérityphlite, «inflammation du tissu cellulaire qui entoure le cæcum », et la péricolite, inflammation du côlon. Le Figaro du même jour parle d'un abcès purulent du gros intestin : " La peau est considérablement inflammée et le pus tend à fuser au-dehors mais il se trouve arrêté par la masse graisseuse très épaisse de l'abdomen " (29 décembre 1882). On apprend que les soins sont compliqués par la corpulence du malade (112 kg), qu'il est atteint d'asthme et de diabète, qu'il souffre de "varices volumineuses à la jambe gauche qui l'ont toujours obligé à porter des bas spéciaux », qu'il a perdu en 1867 l'usage de son œil droit. A la mort de Gambetta, le Petit Parisien se venge de la censure qu'il s'était imposée jusqu'alors : "L'inflammation, jusqu'alors localisée, se répandit sur tout le côté droit, et le pus, s'introduisant dans le sang, le malade fut pris de frissons très souvent renouvelés. [...] Vers six heures du soir, l'inflammation avait fait des progrès effrayants. Plus de doute, l'inflammation psychémique s'étendait, sans limites tracées, dans la fosse iliaque, et allait envahir tout le système circulatoire. [...] A dix heures, $[. .$.$] , la face du malade avait pris une$ teinte terreuse, cadavérique, et des plaques bistres ou verdâtres se montraient sur la peau. Le pus avait déjà envahi l'estomac et la poitrine était le siège de râles muqueux. "C'est fini, [...], je sais que c'est bien fini. Bon courage, mes amis!" Les traits du moribond, livides et horriblement contractés, avaient pris une teinte débile. L'œil vitreux et agrandi exprimait l'angoisse et la souffrance » (le Petit Parisien, 3 janvier 1883).

9. A. Ben-Amos, «Les funérailles de Victor Hugo", in Pierre Nora (éd.), les Lieux de mémoire, vol. 1 : la République, Paris, Gallimard, 1984, p. 473-522. 
Et, le 5 janvier, le journal offre en prime une relation détaillée de l'autopsie : « A l'aide d'une scie à chantrelles, il a scié les côtes sur la partie gauche du tronc. MM. Brouardel et Cornil ont alors extrait les viscères de la cage thoracique. [...] Puis, enfonçant dans l'os frontal le crochet terminant le manche du marteau, il a détaché la calotte crânienne [...]. Le cerveau a été confié à $M$. Mathias Duval, le savant professeur de la Faculté de Paris. Nous croyons savoir que le poids du cerveau de M. Gambetta est supérieur au poids moyen » (le Petit Parisien, 5 janvier 1883). Le cœur, extrait lui aussi, sera conservé et transféré au Panthéon en 1920.

La mort de Gambetta fut un événement politique « énorme » (le Figaro, 3 janvier 1883), qui occupa l'avant-scène de l'actualité jusqu'au lendemain des funérailles nationales, le 6 janvier : "Léon Gambetta est mort. Le patriote qui avait si noblement compris les exigences de l'honneur national et qui avait ramassé le drapeau gisant à terre, pour lui faire affronter encore la pluie des balles. Le républicain qui avait, au bord de la guerre, discipliné son parti pour lui assurer la victoire dans le Parlement et dans le pays. L'homme d'État qui avait enseigné au peuple à résister avec cette seule arme, la loi, aux insurrections du pouvoir. L'admirable orateur. L'ami si sûr et si cordialement simple, cet homme-là est mort. L'heure n'est ni aux panégyriques ni aux longues études » (le Paris, 3 janvier 1883) ${ }^{8}$.

Le journal de Gambetta, la République française, commence alors la publication des innombrables dépêches qui saluent le "patriote illustre, l'espoir de la patrie perdue et le plus éloquent défenseur de la démocratie française » (Comité d'organisation du banquet Hoche de Versailles), «le grand citoyen, l'éminent politique et patriote ardent $»$ (Cercle de Pomerols), « le grand citoyen » (Cercle radical indépendant du Vigan). A Montluc, "le conseil municipal s'est réuni sous l'emprise de la plus profonde et de la plus douloureuse émotion ». « La ville de Paris a ressenti comme la France entière le coup qui vient de la frapper. Gambetta mort, c'est plus qu'une vie d'homme de génie qui s'est éteinte, c'est la République qui se sent blessée dans ce qu'elle a de plus intime. » Défile la liste sans fin de tous ceux qui veulent témoigner, les "francs-tireurs de Châteaudun ", « Démocratie oloronnaise », " le parti républicain de Pont-Audemer ", « la jeunesse des écoles de Grenoble », « l'Association générale des Alsaciens-Lorrains », « un groupe de télégraphistes d'Épinal », « la Ligue de l'enseignement », « la Légion bretonne » et les "francs-maçons de tous les rites $» .$.

Lorsque le conseil municipal de Cambrai affirme que "Gambetta ne sort de ce monde que pour entrer dans l'immortalité », il exprime plus justement l'émotion populaire que l'anticipation dépréciative du Figaro : « Soyez certains que cet homme va paraître plus grand parce qu'il est couché. C'est le phénomène d'optique qu'on constate sur les grands arbres abattus » ( 3 janvier 1883). Le pli de la dénonciation de la dictature gambettiste est trop marqué pour disparaître du jour au lendemain. La presse parisienne va donc se trouver en porte à faux devant les suppressions spontanées de respect et le « succès " des funérailles. Car, au total, c'est bien un procès d'immortalisation républicaine qui s'ouvre alors. Faute de place, il n'est pas possible ici d'analyser les divers aspects du travail politique de célébration et de commémoration. Les contributions que l'apothéose des funérailles nationales ont pu remplir dans la politique mémorielle de la République ont été étudiées ailleurs ${ }^{9}$. Dans le cas de Gambetta, les cérémonies du deuil clôturent, au moins pour un temps, le conflit des interprétations sur le rôle de l'homme politique. "La foule immense », "la houle humaine », "le million de têtes ", "l'énorme quantité de monde » qui s'entasse sur le parcours du cortège funèbre apporte un démenti à tous ceux qui veulent seulement voir dans 


$\begin{array}{lllllllll}\mathrm{D} & \mathrm{O} & \mathrm{C} & \mathrm{U} & \mathrm{M} & \mathrm{E} & \mathrm{N} & \mathrm{T} & \mathrm{S}\end{array}$

la disparition de Gambetta « la fin d'un souvenir » (l'Intransigeant, 3 janvier 1883). Le nombre prouve, physiquement, la gloire de Gambetta, qu'il est incontestablement un «Grand Homme », un fondateur et un héros. La présence des corps constitués, les délégations venues de toute la France, l'apparente émotion du «peuple » de Paris matérialisent la réalité d'une légitimité. Mais la sanctification de Gambetta n'était pas acquise d'avance.

En réponse à l'éditorial de la République française, les journalistes reprennent le mot d'Anacharsis Klootz, «France, guéris-toi des individus ». Le Petit Parisien dénonce la théorie des «hommes providentiels et des sauveurs » (3 janvier 1883) et divise la vie de Gambetta en deux parts: «Pendant la première, M. Gambetta a vaillamment servi la République; pendant la seconde, par une suite d'actes que l'histoire condamnera, il l'a compromise » (le Petit Parisien, 3 janvier 1883). L'image souffre de sa réputation d'autoritarisme : «C'est précisément parce que, malgré les dangers de sa politique autoritaire, malgré ses coups de tête, malgré son mépris pour la légalité, malgré ses créatures prétoriennes, M. Gambetta était un grand patriote, que la France, une nation reconnaissante et généreuse, fait de sa mort un deuil national » (le Petit Journal, 5 janvier 1883). Les journalistes, après l'hommage obligé au «vrai fondateur de la République ", préfèrent se réfugier dans la célébration du régime. «C'est le privilège du régime fondé sur le droit populaire de défier l'instabilité des personnes et des choses. Nos idées sont tout parce qu'elles sont immortelles. Les hommes qui les servent ne sont rien que des instruments éphémères » (le Petit Journal, 2 janvier 1883). « Dans une démocratie, les hommes peuvent disparaître, les institutions demeurent. L'époque est passée où les destinées de la patrie se trouvaient dans les mains d'un seul. L'idée qu'il y a des hommes nécessaires est une idée monarchique dont la France s'est débarrassée depuis long- 
temps» (le Petit Parisien, 3 janvier 1883). Le Gambetta des dernières années est ainsi systématiquement contourné au profit de la République et de la France éternelle : "Audessus des hommes qui passent, au-dessus des partis qui luttent, il y a la France immortelle, la France libérale, civilisatrice, laborieuse et pacifique » (la Liberté, 3 janvier 1883). Comme souvent, l'adversaire s'interdit de continuer la lutte par-delà la mort. Le respect de l'ennemi mort constitue une des formes instituées « de l'expression obligatoire des sentiments »: "Vivant, nous l'avons combattu. Mort, nous nous abstiendrons de ne rien dire contre sa mémoire »(le Soir, 3 janvier 1883). Certains, au nom d'une même conception du métier, rendent hommage à son savoir-faire : «Il s'était levé contre Dieu. Il est tombé. [...] Avec lui, quoi qu'on en dise, la République est clouée dans le cercueil. [...] Certes, il avait des défauts, il avait des vices. Mais c'était un rude manieur d'hommes, un remueur de foules, et quand il n'y a plus à le craindre, ce serait puéril de la rabaisser. Nous ne le ferons pas » (Paul de Cassagnac, le Pays, 3 janvier 1883, et le Petit Caporal, 3 janvier 1883). Paradoxalement, ce sont les quotidiens socialistes, comme la Bataille ${ }^{10}$ ou la Lanterne et, plus encore, les journaux de la presse républicaine conservatrice, le XIX ${ }^{e}$ siècle, le Temps, ou le Journal des débats, qui témoignent, au travers de leur laconisme volontaire, de la grandeur passée de l'homme : "Ce que Thiers fut pour la France, Gambetta le fut pour la République. C'est pourquoi son nom restera, indissolublement, lié à la fondation du gouvernement nouveau de la France. Après Thiers, la France est restée, après Gambetta, la République restera » (le Journal des débats, 3 janvier 1883). Et E. Scherer, dans un parallèle avec Mirabeau qui s'impose comme une évidence, lui reconnaît le mérite de l'action transactionnelle, de la politique positive, la gloire d'avoir su « faire réussir la République en l'astreignant à un pas régulier, assurer l'avenir de la démocratie en la prélevant des chimères, rendre la révolution définitive en la purgeant de l'esprit révolutionnaire " (le Temps, 5 janvier 1883).

Si le texte de Lannelongue mérite publication, ce n'est évidemment pas à la typhlite qu'il le doit, mais à la personne du patient, cet homme qui retenait en lui la fondation de la République et quelque chose de l'histoire de France. Des circonstances très particulières semblent être requises pour que le territoire de l'intimité, la face cachée d'une homme public, même hors du commun comme Gambetta, puisse être aussi totalement mis à nu, approprié $^{11}$. Et d'abord, l'exposition détaillée de ses souffrances constitue le prix à payer pour rembourser la dette de sens ouverte par sa vie privée. Pour le bien de la République, tout doit être montré. On voit aussi que la divulgation du rapport apporte, par anticipation, une réponse à une possible mise en cause des médecins, et que ces derniers ont, dans cette affaire, également intérêt à lever le secret thérapeutique car, de la publicité, dépend leur légitimité scientifique et sociale, et par ricochet, l'autorité du corps médical tout entier. Le texte témoigne à la fois de la compétence et du dévouement des médecins et apporte la démonstration de l'indépendance de la profession à l'égard des pouvoirs.

Sur ces nécessités se greffent d'autres raisons qui, à leur tour, commandent l'énonciation. Les analyses de P. Ariès sur la mort de la mort et de N. Elias sur l'économie des habitus et les formes historiques de la sensibilité $^{12}$ sont trop connues pour qu'il soit nécessaire de les rappeler. Indiquons seulement que le seuil de sensibilité aux profanations de l'intimité s'est considérablement élevé depuis la fin du XIX ${ }^{\mathrm{e}}$ siècle. En outre, la langue médicale, fonctionnellement libérée des contraintes de la civilité, apparaît, alors même qu'il s'agit d'un discours mondain, affranchie des règles élémentaires du $\operatorname{tact}^{13}$. Joue enfin l'incertitude d'un savoir qui hésite, en ces temps pré-pastoriens, sur la nature du signe et du symp- 


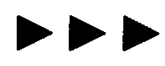

14. Michel Foucault, Naissance de la clinique, Paris, Puf, 1972. Cf. aussi le beau texte «Traces" de Carlo Ginzburg (Mythes, emblèmes, traces, morphologie et histoire, Paris, Flammarion, 1989).

15. Norbert Elias, la Solitude des mourants, Paris, Christian Bourgois éditeur, 1987, p. 14.

16. Ernst H. Kantorowicz, les Deux Corps du roi, Paris, Gallimard, 1989 ; Ralph E. Giesey, Le roi ne meurt jamais, Paris, Flammarion, 1987 ; Michel Vovelle, Mourir autrefois, Paris, Gallimard, 1974. tôme ${ }^{14}$ et qui, dès lors, inscrit l'enregistrement clinique dans une logique de l'exhaustivité. Parce qu'il s'agit de Gambetta, parce que la médecine porte une attention infinie aux signes, parce qu'il faut jouer le jeu de la vérité, Lannelongue s'attarde sur l'" empâtement intestinal », les gaz et « éructations fréquentes », les urines " très épaisses », " de couleur betterave et jumenteuses", les «garde-robe abondantes ", la « langue saburrable, les « efforts de vomissements ». " 16 décembre : pendant le pansement, M. Gambetta est tourmenté par d'assez violentes coliques, il a des renvois incessants ; sa figure est rouge, son ventre un peu tendu ». "Choses » auxquelles la science actuelle n'accorde plus guère d'intérêt. «Choses » aussi que le code de conduite actuel interdit, sous peine de vulgarité, sous peine d'atteinte à la personne, d'évoquer, et qui précisément étonnent parce qu'elles sont là exprimées sans les précautions aujourd'hui en usage.

Mais ce n'est pas tout. Reste un dernier aspect, qui n'est pas le moindre pour comprendre la résonance de ce document. D'ordinaire, la pratique médicale réduit l'individu à des organes et des fonctions, la langue objective la personne en un corps. Or ici, elle laisse ouvert l'accès à la particularité, à l'humain. Le discours, lavé, pour l'occasion, de la plupart des termes savants qui habituellement obscurcissent le sens et en même temps brisent le rapport à l'autre, autorise l'identification. La maladie est incarnée dans un corps dont on res-' sent la fatigue, les malaises, les gênes, comme ce mouvement de la jambe droite qui la ramène spontanément en position fléchie. Léon Gambetta, dans cette chronique de la mort, reste une personne, et s'il reste un être humain, c'est parce que Lannelongue ne dissocie pas la maladie de la souffrance. Bref, ce qui confère à ce texte son ultime singularité, et en définitive sa force, c'est qu'il s'attache autant à la peine humaine qu'à la santé des organes. Comme si la personnalité de Gambetta le sau- 
vait de l'objectivité. Alors, humain trop humain, Gambetta, dans ce drame universel et singulier, apparaît là comme une figure anonyme de l'humanité, et c'est moins l'individu Gambetta qui nous retient que Gambetta en tant qu'individu, un être quelconque et, au fond, nous-mêmes dans notre relation à la douleur, à la maladie et à la mort. La mort est l'affaire exclusive de ceux qui sont en vie. $\mathrm{N}$. Elias le note en des termes très simples au début de Uber die Einsamkeit der Sterbenden: «La mort [écrivait-il] est un problème des vivants. Morts, les êtres humains n'ont pas de problèmes ${ }^{15}$." Précisément, le fait de mourir constitue «un problème » pour ceux qui vivent, parce que, malgré Orphée et ses frères innombrables, malgré le Ivan Illitch de Tolstoï, il est impossible de savoir (et de partager), il est impossible de penser (et de vivre) la mort du point de vue des morts.

Le mal, ici, se signale par une "vive douleur dans le flanc droit ", plus forte qu'à l'accoutumée et dont Gambetta s'inquiète un peu, un mouvement de son corps inconnu. Très vite se multiplient les signes pathogènes. L'inflammation rapidement se développe, et se découvre en même temps l'impuissance de la médecine. Le «tympanisme » persistant de la paroie abdominale, le grossissement des ganglions inguinaux, l'apparition d'un érysipèle s'inscrivent tour à tour dans le tableau nosographique de la typhlite « que paraissait rendre indéniable la constatation d'un engorgement péricæcal ». Sur la fin, les médecins notent le calme du malade, à la fois signe d'un état psychologique, l'indifférence à toutes choses, adieu déjà prononcé au monde, et symptôme morbide qui prend place à côté du froid qui envahit les extrémités, du pouls qui faiblit, de la respiration qui ralentit.
«Dimanche 31 décembre, huit heures. Nuit calme et dans l'affaissement jusqu'à cinq heures du matin. A ce moment, M. Gambetta est pris d'un délire léger, qui reparaît à plusieurs reprises [...] un peu plus tard, il a le hoquet pendant quelques instants. La faiblesse est grande; il n'éprouve d'ailleurs aucune souffrance. On lui donne du café, il le rejette ; on recommande l'usage du vin de Champagne et l'emploi plus continu de l'eau-de-vie et du rhum. Une heure - la physionomie du malade est calme, mais le visage présente une teinte légèrement violacée... La cavité buccale est extrêmement sèche, il répond avec difficulté tant qu'il n'a pas humecté sa bouche [...]. Vers deux heures, les parties qui sont hors du lit, les mains surtout deviennent fraîches. Le pouls oscille entre 120 et 140 et par temps il a quelques irrégularités; le nombre de respirations est de 38 à 40. L'état du ventre est toujours le même, l'érysipèle semble éteint. Le vin de Champagne est mal toléré. Dix heures du soir : les symptômes alarmants se sont multipliés et s'aggravent. Le malade a cependant encore sa connaissance et il répond un dernier mot à onze heures moins un quart. Le dénouement est imminent et la mort arrive sans secousse quelques minutes avant minuit. » Avant que ne commence la nouvelle année, 1883.

Depuis longtemps déjà, l'humanisation des récits de mort n'était due qu'aux grands ${ }^{16}$. C'est donc à plusieurs titres que Gambetta gagne son immortalité, par sa vie, par le triomphe de ses funérailles mais aussi, plus subtilement, par la relation d'une mort qui, pour partie par amitié, pour partie par déférence à l'égard du « Grand Homme », n'abolit jamais la personne. 


\section{Extraits de «Blessure et maladie de M. Gambetta», le Concours médical, $n^{o} 4,1883$.}

Nous laissons d'abord de côté tout ce qui a trait à la blessure de M. Gambetta ; il est surabondamment démontré que le grand orateur s'était blessé lui-même par imprudence le 7 novembre, et que la plaie, ainsi produite, était en bonne voie de cicatrisation le 9 décembre, bien que le projectile eût coupé l'arcade palmaire superficielle, légèrement atteint l'artère orbitale et incomplètement coupé le nerf cubital.

C'est seulement à partir du 8 décembre que M. Gambetta se plaint d'avoir le ventre distendu par des gaz. Le 9, on constate du dégoût pour les aliments. « Le 10, le malade nous apprend que la veille au soir, en faisant des efforts pour aller à la garde-robe, il a ressenti subitement une vive douleur dans le flanc droit, dont il précise mal le siège. Cette douleur a déterminé de l'insomnie, et le dimanche il s'en plaint encore, quoiqu'elle soit beaucoup moins accentuée. L'état saburral est plus prononcé, l'inappétence est complète.

«M. le professeur Charcot, qui l'a vu dans la journée, lui a conseillé un lavement purgatif. L'exament du ventre ne révèle rien d'anormal ; il n'y a nulle part d'empâtement, le siège de la douleur est très vague, et $\mathrm{M}$. Gambetta se plaint à peine quand on presse fortement dans le flanc ou dans la région lombaire. On réveille pourtant de la sensibilité sur la paroi latérale et inférieure du thorax du côté droit ; il est proposé d'appliquer sur ce point un sinapisme ; mais, comme on a déjà pratiqué un large badigeonnage de laudanum, M. Gambetta ne paraît pas disposé à accepter le sinapisme, et il ajoute qu'il ne souffre pour ainsi dire plus. »

Le 11, même état; purgation efficace ; on permet au malade de se mettre dans un fauteuil, et de fumer un cigare. Le 12, M. Gambetta a déjeuné de très bon appétit. Le 13, le ventre paraît libre. Le 14, le malade circule dans la maison. Le 15, malaise abdominal, éructations fréquentes. M. Gambetta se promène pendant vingt minutes dans son jardin. T. 36,6. Le 16, malgré des douleurs abdominales assez vives, il fait avec le plus grand plaisir une promenade en voiture; il déclare en éprouver un réel bien-être ; mais, peu de temps après, les malaises reparaissent et à huit heures du soir, on trouve une température de $39^{\circ}, 6$, avec un poul à 88 . A dix heures, à l'arrivée de $M$. Lannelongue, mandé en toute hâte, « $M$. Gambetta ressent une grande chaleur; il est en pleine transpiration. L'examen de la poitrine ne révèle rien ; tous les phénomènes sont concentrés dans le ventre, qui est tendu et un peu douloureux à la pression du côté droit ; on n'y trouve pourtant pas d'empâtement.

«Le 17, M. Siredey, après avoir procédé à un examen complet du malade, rejette l'hypothèse de toute complication thoracique. Ayant constaté un empâtement douloureux et très circonscrit dans la fosse iliaque droite, il me transmet une note, dans laquelle je lis cette phrase : "Je crois que la typhlite est ce qu'il y a de plus probable." A ce moment, la température est encore élevée, et $M$. Gambetta ressent les mêmes symptômes de tension abdominale et d'éructation. Le régime prescrit comprend exclusivement des boissons : limonade tartrique, grogs et bouillons.

[...] « Le lundi 18 décembre, [...] toute l'attention doit se concentrer désormais sur les accidents qui ont pour point de départ la cavité abdominale, et rendez-vous est pris avec M. Siredey pour que nous ayons le lendemain une conversation à ce sujet. Aujourd'hui, d'ailleurs, la tuméfaction persiste malgré la purgation de la veille, qui a produit trois évacuations abondantes. M. Gambetta est fatigué et cherche à reposer.

«A six heures moins un quart, il se produit pour la première fois un frisson assez intense de vingt-cinq minutes de durée, suivi d'une forte impression de chaleur et de quelques efforts de vomissement [...].

«Le mardi 19, nous nous réunissons avec M. Siredey pour visiter le malade à huit heures du matin. Il a eu dans la nuit un nouveau frisson très intense d'une demi-heure de durée, suivi d'une forte chaleur, d'une évacuation d'urine abondante et aussi d'une transpiration considérable [...]. L'examen attentif de la cavité abdominale donne les résultats suivants : le ventre est souple et d'un aspect uniforme, l'exploration de la fosse iliaque droite est facile et fort peu douloureuse 


\section{$\begin{array}{lllllllll}\mathrm{D} & \mathrm{O} & \mathrm{C} & \mathrm{U} & \mathrm{M} & \mathrm{E} & \mathrm{N} & \mathrm{T} & \mathrm{S}\end{array}$}

superficiellement; on constate dans sa partie la plus élevée, à deux travers de doigt environ au-dessus de l'épine iliaque supérieure, un empâtement très profond et douloureux à la pression, de forme allongée et cylindrique, ressemblant à un boudin. Cet empâtement suit le trajet du colon ascendant et cesse d'être senti au-delà d'une longueur de quatre à cinq centimètres environ. La percussion en révèle aussi l'existence; il y a là une submatité circonscrite, séparée de la matité du foie par une zone transversale sonore d'un pouce environ ; l'inspection de ce dernier organe permet de le considérer comme sain et plutôt d'un petit volume. En explorant la région lombaire, on ne découvre rien d'anormal ; une pression forte au niveau du rein ne réveille pas de sensibilité. Les mouvements du membre inférieur de ce côté sont tout à fait libres. Les urines examinées avec soin révèlent l'existence d'une assez forte proportion d'albumine, elles sont très épaisses, de couleur betterave et jumenteuses.

« Nous eûmes avec M. Siredey un long entretien qui nous amena à conclure à l'existence d'une pérityphlite que paraissait rendre indéniable la constatation d'un engorgement péricæcal.

"Régime lacté, boissons fraîches, limonade et eau minérale ; 1 gramme de sulfate de quinine dans la journée.

$[\ldots]$

Mercredi 20. "Huit heures du matin. - Visite de MM. Siredey et Lannelongue. La nuit a été excellente, le sommeil prolongé. M. Gambetta se trouve très bien, il ne souffre plus du ventre; l'examen que nous en faisons ne révèle que de la sensibilité à une pression assez forte toujours dans le même point; l'état local a la même apparence que la veille. La quantité des urines rendues est normale, elle était moindre hier ; elles sont beaucoup plus limpides et toujours albumineuses.

«Régime lacté, quelques bouillons, eau rougie, un gramme de sulfate de quinine dans la journée.

«A deux heures de l'après-midi, frisson assez intense, longue période de chaleur suivie de sommeil, transpiration moins abondante. Pendant le frisson, vomissement du grog ingéré. Dans la soirée, le malade se trouve bien, il ne se plaint aucunement, il a eu d'assez longs moments de sommeil et quelques bourdonnements d'oreille provoqués par la quinine.

«En dehors de nos conversations du matin et du soir, nous eûmes souvent à Paris de longs entretiens avec M. Siredey sur la situation de M. Gambetta. Elle nous occupa une partie de la soirée de ce jour. Le fait de l'existence d'une pérityphlite ressortit de notre discussion comme la donnée la plus certaine ; mais le mode d'invasion, l'intensité des frissons et des accès fébriles auxquels succédait une chute de la température jusqu'au degré normal et une rémission complète, le bien-être du malade dans les intervalles apyrétiques ne nous parurent pas suffisamment en harmonie avec l'idée d'une inflammation franche, légitime, d'un type régulier. Pour la première fois, nous parlâmes d'une perforation extra-péritonéale de l'intestin comme cause première des accidents. [...]

Le 22. T. mat. $39^{\circ}, 5$, à quatre heures; à huit heures, $36^{\circ}, 8$; soir $37^{\circ}$.

« Le malade se trouve très bien et nous parle de l'excellente nuit qu'il a passée. Notre examen nous fait reconnaître un ballonnement du ventre plus marqué que les jours précédents. La pression est plus douloureuse que la veille, et nous observons que l'empâtement descend encore vers l'épine iliaque supérieure, tout en restant profond et séparé de la paroi abdominale par une zone sonore ; [...]

Samedi 23.

T. mat. $36^{\circ}, 2$; soir $38^{\circ}$.

« Il exprime le désir de voir M. le professeur Charcot; la réunion a lieu à huit heures du matin. La fin du jour précédent et la nuit ont été très bonnes. M. Gambetta a longuement dormi. M. Charcot trouve un état général dans de bonnes conditions, la physionomie favorable, la langue humide. Le ventre étant moins distendu par les gaz, l'exploration de la fosse iliaque est facile, et $M$. Charcot reconnaît que la partie inférieure et interne est libre ; il n'en est pas de même en dehors et en haut, où existe un empâtement qui occupe le cæcum et la partie inférieure du côlon ascendant; c'est la portion postérieure de ces organes qui semble atteinte, ainsi que le 
tissu graisseux sur lequel ils reposent. Actuellement, selon M. Charcot, l'affection serait une pérityphlite primitive se propageant sur le côlon, et il prononce le nom de péricolite concomitante. Il n'y a aucun indice de suppuration, ni œdème, ni fluctuation, ni douleurs spontanées. L'opinion du professeur Charcot confirme et précise le diagnostic posé par les médecins ordinaires.

[...]

Le 24 et le 25 : Assez bonnes journées, la température varie de $36^{\circ}, 8$ à $38^{\circ}, 6$. Lavement purgatif, œuf frais, lait et bouillon. Il n'y a pas eu de frisson depuis le 22.

Le 26, T. $38^{\circ}$, P. $76,-38^{\circ} 2$ P. 80 .

[...]

«Le ventre présente un tympanisme prononcé qui gêne le malade depuis quelques moments : sur la place occupée par le vésicatoire existe une inflammation de la peau assez prononcée avec rougeur et œdème, (c'est la première fois qu'on constate ce phénomène nouveau, mais il perd de sa valeur clinique, car il n'existe qu'à la place même du vésicatoire). [...] Les ganglions inguinaux sont douloureux. La pression au niveau du rein ne réveille pas de douleur; M. Gambetta a souffert spontanément dans le côté, il est un peu affaissé. Un lavement pris le soir amène une évacuation. "

Le 27 , T. mat. $38^{\circ}$, P. 80 ; soir, $39^{\circ}$, P. 100.

«La nuit a été un peu agitée et le sommeil très interrompu. Le malade accuse quelques douleurs superficielles dans le côté, dans la racine du membre et jusque dans la jambe ; il tient plus volontiers le membre inférieur droit fléchi sur le bassin et dans la rotation en dedans. Quand on lui demande d'étendre ce membre, il le fait sans douleur, mais il le ramène dans la flexion; il y a incontestablement un certain degré d'irritation du psoas. Même état local qu'hier, pas de fluctuation, subsonorité sur la paroi latérale correspondante à l'engorgement. La surface du vésicatoire est rosée et œdémateuse, on voit quelques traînées qui vont vers le pli de l'aine. Le malade a pris du chocolat au lait à son déjeuner, du lait et deux grogs dans la journée. Le soir, évacuation après un lavement purgatif. »

Le jeudi 28, consultation de MM. Charcot, Verneuil, Trélat, Siredey, Gilles, Fieuzal et Lannelongue. T. mat. $38^{\circ}$, P. 80 . Soir, $38^{\circ}, 8$, P. 100.

«Matin. - Le malade a passé une bonne nuit et il se sent reposé : il prend deux verres d'eau purgative à huit heures qui amènent dans la journée une évacuation abondante de matières liquides et de gaz. Le régime alimentaire s'est composé de lait, de vin et de grogs. A cinq heures du soir a lieu la consultation. Les médecins réunis, après avoir discuté toutes les hypothèses que pouvait suggérer l'état du malade, furent unanimement d'accord sur les conclusions suivantes :

«L'existence de la pérityphlite est incontestable ; toute autre hypothèse doit être écartée, les probabilités en faveur d'une suppuration autour du gros intestin, dans le tissu cellulo-graisseux sur lequel il repose sont très grandes. Les résultats fournis par la recherche attentive de la fluctuation étaient absolument négatifs, il n'existe en aucun point de collection purulente. Peut-être y a-t-il une infiltration de pus ? La sonorité intestinale déborde de toutes parts, même en arrière, l'empâtement profond.

«Ces conditions réunies interdisent une intervention chirurgicale qui serait pleine de périls sans donner aucun espoir fondé d'un résultat favorable. »

Le 29, T. mat. $36^{\circ}, 8$ P. 100 . T. soir. $38^{\circ}, 7 . \mathrm{P}, 108$.

«L'expression faciale est calme, mais la langue est sèche pour la première fois, la peau est fraîche, le ballonnement du ventre est toujours prononcé et le malade a eu deux évacuations dans la journée. L'examen local montre un érysipèle fort étendu, couvrant la paroi latérale droite de l'abdomen et le tronc du même côté depuis l'angle inférieur de l'omoplate jusqu'à la racine de la cuisse, qui est aussi envahie en arrière ; [...]

Samedi 30 , T. mat. $37^{\circ}, 7$. P. 108 . Soir, $38^{\circ}, 6$, P. 110.

«La nuit a été mauvaise et le sommeil interrompu sans qu'il y ait eu cependant du délire. La bouche est amère et la langue sèche, la peau moite ; le malade a pris sa potion au quinquina, 
mais il a vomi la dernière cuillerée ; la rougeur de l'érysipèle est moindre et le gonflement de la peau peu accusé, le ventre est aussi plus souple. M. Gambetta ne paraît pas inquiet, il semble moins absorbé qu'hier et nous parle de l'insomnie de la nuit ; la parole est facile, mais la voix est moins forte, et le nombre des respirations s'élève à trente-quatre par minute. Thé au lait, lait additionné de kirsch, grogs.

«Quatre heures du soir. - Consultation de MM. Charcot, Verneuil, Trélat, Siredey et Lannelongue, M. Paul Bert était présent. Pendant la journée, M. Gambetta s'est montré indifférent à toutes choses, il a eu quelques moments de sommeil ; il n'a ressenti aucune douleur, il est toujours gêné par les gaz et a eu un vomissement.

«Les médecins qui ont pris part à la consultation donnent successivement leur avis. D'un commun accord, ils reconnaissent que la situation s'est considérablement aggravée et qu'aucune opération n'est indiquée, ni possible. Ils considèrent que les seules indications à remplir sont relatives à l'état fébrile et à la nécessité de soutenir les forces du malade.

« Dimanche 31, T. mat. 37, P. 120. R. 40.

«Huit heures. - Visite de M. Siderey [sic]. Nuit calme et dans l'affaissement jusqu'à cinq heures du matin. A ce moment, M. Gambetta est pris d'un délire léger, qui reparaît à plusieurs reprises jusqu'à sept heures et demie ; un peu plus tard, il a le hoquet pendant quelques instants. La faiblesse est grande; il n'éprouve d'ailleurs aucune souffrance. On lui donne du café, il le rejette ; on recommande l'usage du vin de Champagne et l'emploi plus continu de l'eau-de-vie et du rhum.

«Une heure. - Visite de M. Lannelongue. La physionomie du malade est calme, mais le visage présente une teinte légèrement violacée apparente sur les joues, le nez et les oreilles ; la cavité buccale est extrêmement sèche et, quand on adresse la parole au malade, il répond avec difficulté tant qu'il n'a pas humecté sa bouche; du reste, M. Gambetta possède toute sa lucidité et, jusqu'à quatre heures, il ne se plaint d'aucune souffrance. Vers deux heures, les parties qui sont hors du lit, les mains surtout, deviennent fraîches. Les [sic] pouls oscille entre 120 et 140 et par temps il a quelques irrégularités; le nombre des respirations est de 38 à 40 . L'état du ventre est toujours le même, l'érysipèle semble éteint.

«Le vin de Champagne est mal toléré ; il est recommandé de ne plus employer que le thé fortement additionné de rhum, les grogs à l'eau-de-vie, et de réchauffer le malade avec des boules d'eau chaude.

« Dix heures du soir. - M. Lannelongue. Les symptômes alarmants se sont multipliés et s'aggravent, le malade a cependant encore sa connaissance et il répond un dernier mot à onze heures moins un quart. Le dénouement est imminent et la mort arrive sans secousse quelques minutes avant minuit. " 